\title{
CORRELATION BETWEEN COGNITIVE FUNCTION WITH DISEASE ACTIVITY OF SYSTEMIC LUPUS ERYTHEMATOSUS PATIENTS IN DR. HASAN SADIKIN HOSPITAL BANDUNG: AN ANALYTICAL CROSS-SECTIONAL STUDY
}

\author{
Aep Saepudin,1 Paulus Anam Ong,, Syarief Hidayat, ${ }^{3}$ Andri Reza Rahmadi, ${ }^{1}$ Laniyati Hamijoyo' \\ ${ }^{1}$ Department of Internal Medicine, Faculty of Medicine, Universitas Padjadjaran/Dr. Hasan Sadikin General Hospital, \\ ${ }^{2}$ Department of Neurology, Faculty of Medicine, Universitas Padjadjaran/Dr. Hasan Sadikin General Hospital \\ ${ }^{3}$ Department of Cardiology and Vascular Medicine, Faculty of Medicine, Universitas Padjadjaran/Dr. Hasan Sadikin General \\ Hospital
}

\section{A R T I C L E I N F O}

\section{Keywords:}

Cognitive dysfunction

MoCA-Ina

Systemic lupus erythematosus

SLEDAI-2K

\section{Corresponding author: \\ Laniyati Hamijoyo \\ E-mail address: \\ hamijoyo@yahoo.com}

All authors have reviewed and approved the final version of the manuscript.

https://doi.org/10.37275/IJR.v10i1.1

\begin{abstract}
A B S T R A C T
Background: Cognitive dysfunction was found in 55-80\% Neuropsychiatry Systemic Lupus Erythematosus (NPSLE) patients. Serious concern from clinicans was needed as its impact to patient's quality of life. Disease activity is expected to be affecting patient's cognitive function. Previous studies regarding correlation between disease activity and cognitive dysfunction showed various results. This study aimed to evaluate the correlation between disease activity and cognitive function in SLE patients. Methods: This study is an analytical cross-sectional study. Subjects were SLE patients at the rheumatology clinic of Dr. Hasan Sadikin Hospital Bandung during June-August 2017. Subject's evaluations included disease activity assessment using SLE disease activity index-2K (SLEDAI-2K) and cognitive function assessment using MoCA-Ina test. Data were analyzed by using Spearman Rank correlation test. Results: Mean age of the subjects was $31 \pm 8$ years old, most of them were senior high school graduates $(65.8 \%)$ and median length of study was 12 years. Subject's median duration of illness was 44 months. Their MoCA-Ina median score was 25, while SLEDAI-2K median score was 6 . Cognitive dysfunctions were found in more than half of subjects $(52.63 \%)$, which memory domain $(78.95 \%)$ was most frequently impaired. Most of subjects were patients with active SLE (63.2\%). Correlation test showed there was no correlation between SLEDAI-2K score and MoCA-Ina score $\left(r_{s}=0.023, p=0.445\right)$. Conclusion: There was no correlation between disease activity (SLEDAI-2K score) and cognitive function (MoCA-Ina score).
\end{abstract}

\section{Introduction}

Systemic Lupus Erythematosus (SLE) mostly attacks women at in their reproductive age along with high mortality rates. Incidence of SLE was almost three times higher over the last four decades and it is mostly caused by earlier detection and improvement in diagnosing of SLE. ${ }^{1,2}$ SLE disease course is marked by an exacerbations and remissions, hence careful monitoring of the disease activity is needed. A comprehensive management including frequent assessment of disease activity may enable us to control disease's progressivity, prevent its complications, and provide optimal therapies to each patient. ${ }^{3}$ SLE disease activity can be evaluated by using Systemic Lupus Erythematosus Disease Activity Index-2000 (SLEDAI$2 \mathrm{~K}$ ), which is the most common instrument used to evaluate disease activity in clinical practice and research, as it proved to be valid and sensitive.4-6 SLEDAI-2K represents immunological and inflammatory state in the nine organ systems that involved in NPSLE, such as kidney, hematology, musculoskeletal, mucocutaneous, eyes, vasculitis, serositis and immunology. Higher SLEDAI-2K score 
shows the disease activity is more severe and more organ systems involved.7-8

SLE is an autoimmune disease with a broad spectrum of clinical manifestations and involving multiorgan systems, including Neuropsychiatric Systemic Lupus Erythematosus (NPSLE), which contributes to increased mortality and morbidity, also decreased quality of life in SLE patients. Cognitive dysfunction had highest prevalence, and was found in 55 to $80 \%$ of NPSLE patients. Pathogenesis of cognitive dysfunction in SLE was still unknown, however several clinical association have been reported its correlation with cognitive dysfunction such as:9 (1) There was a correlation between decreased cognitive function and active NPSLE or previous NPSLE, (2) Psychological distress that affect cognitive performance, (3) There was correlation between cognitive impairment and some certain autoantibodies in the serum or cerebrospinal fluid or both. Possible roles for antibodies such as antiphospholipid and anti-NMDAR in the pathogenesis of cognitive dysfunction have been suggested, (4) In SLE patient with cognitive dysfunction, the cytokines (IL-1, IL-6, IL-10 and IF- $\gamma$ ) were increased in the hippocampus area.

Several factors were expected to have an impact on cognitive dysfunction in SLE such as vascular injuries, neuron injuries caused by autoantibodies and cytokines, and metabolic effects. Antibodies that play roles in the pathogenesis of cognitive dysfunction were antiphospholipid antibody and anti-NMDAR. In hypercoagulable state, antiphospholipid antibodies lead to ischemia in the brain tissue and anti-NMDAR antibodies are likely to cause injuries in the hippocampus, areas associated with learning process. ${ }^{10,11}$ Cytokines (IL-1, IL-6, IL-10 and IFN- $\gamma$ ) involved in cognitive dysfunction. Previous studies showed the excessive expression of cytokine genes in the hippocampus. Those pro inflammatory cytokines could induce apoptosis of the neurons and are thought to drive autoantibody production in SLE.12,13 Cytokines are predisposing neuronal growth, differentiation and development, also causing changes in receptor phosphorylation in the cellular level, increasing intracellular calcium level, and disrupt millions of signal transduction cascades which affecting neuronal plasticity. In SLE, cytokines (IL-1, IL-6, IL-10 and IF- $\gamma$ ) were involved in induction and amplification process of the inflammation, also showed their influences to memory and learning function. ${ }^{13}$

Our previous study showed that cognitive dysfunction was found in 50\% SLE patients, as memory domain was the most frequently impaired among other cognitive domains. ${ }^{10}$ In other study, most of cognitive domains were memory impairment, attention deficits, problem solving difficulties, poor decision making abilities. An early screening is needed as cognitive dysfunction does have an impact to patients's quality of life, decreased work productivity and patient compliance. ${ }^{14,15}$

Indonesian version of Montreal Cognitive Assessment (MoCA-Ina) has been validated and commonly used as a rapid screening instrument for detecting cognitive dysfunction in Indonesia. It also has high sensitivity and specificity to assess cognitive function in adult. MoCA-Ina assesses multiple cognitive domains including attention and concentration, excecutive function, memory, language, visuospatial skills, conceptual thinking, and orientation. 16,17

Regarding therapy and prognosis, correlation between disease activity and cognitive function is remarkable, however former studies showed various results. ${ }^{18-21}$ This study aimed to evaluate the correlation between SLE disease activity based on SLEDAI-2K score and cognitive function based on MoCA-Ina score in SLE patients.

\section{Research Methods}

This is an analytical cross-sectional study. Subjects were SLE outpatients at the Rheumatology Clinic of Dr. Hasan Sadikin Hospital Bandung during June to August 2017. The evaluation included disease activity assessment using SLE disease activity index-2K (SLEDAI-2K) and cognitive function assessment using MoCA-Ina test.

Consecutive data collection was carried out at Rheumatology Clinic and Memory Clinic Dr. Hasan Sadikin Hospital Bandung from June to August 2017. 
Subjects who fulfilled criteria of anxiety disorder or moderate to severe depression using Beck anxiety inventory (BAI) and Beck depression inventory (BDI) questionnaires were excluded from this study. All eligible subjects were evaluated by using SLEDAI-2K score for disease activity and MoCA-Ina test for cognitive function. Data were analyzed using Rank Spearman correlation test.

\section{Results}

Mean age of the study subjects was 31 years old, most of them were senior high school graduate or equals $(65.8 \%)$ with median length of study was 12 years. Most subjects (68.4\%) work as housewives. Subject's median disease duration was 44 months. Their cognitive function based on MoCA-Ina median score was 25, while disease activity based on SLEDAI-2K median score was 6.

Patient was considered having cognitive dysfunction if MoCA-Ina scores less then 26. Table 2 showed that most subjects (52.63\%) had cognitively impaired. Based on impaired cognitive domain, memory domain (78.95\%) was most frequently impaired, followed by executive function (68.42\%). There were subjects who had low memory score despite the fact that they had normal total cognitive score $(\geq 26)$. Hence, there were more subjects with memory impairment than subjects with global cognitive dysfunction.

According to statistical assessment using Chi Square Test found cognitive dysfunction both in active or inactive SLE and in severe disease activity or mild to moderate disease activity were not statistically significant $(\mathrm{p}>0.05)$.

Figure 1 showed correlation analysis by using Rank Spearman test with correlation coefficient 0,023 and $\mathrm{p}=$ $0,445(p>0,05)$, thus there were no correlation between disease activity and cognitive function.

Table 5 and Figure 2 showed correlation analysis by using Rank Spearman test, results were: (1) There was a negative correlation tendency between age and cognitive function based on MoCA-Ina score $\left(\mathrm{r}_{\mathrm{s}}=-0.230\right.$, $\mathrm{p}=0.083$ ), suggesting that the older the subjects were, the lower the MoCA-Ina score obtained, (2) There was no correlation between disease duration and cognitive function based on MoCA-Ina score, (3) There was a moderate positive correlation between subject's length of education and cognitive function based on MoCA-Ina score $\left(r_{s}=0.436, p=0.003\right)$, suggesting that the longer the length of education was, the higher MoCA-Ina score obtained.

Table1. Characteristics of the subjects

\begin{tabular}{ll}
\hline Characteristic & $\mathrm{n}=38$ \\
\hline Age (years), mean \pm SD & $31 \pm 8$ \\
Level of education, n (\%) & \\
$\quad$ - Elementary school & $2(5,3)$ \\
- Junior high school & $7(18.4)$ \\
- Senior high school & $25(65.8)$ \\
- Diploma (D1) & $2(5.3)$ \\
- Bachelor (S1) & $2(5.3)$ \\
Length of study, median (min - max) & $12(6-16)$ \\
Occupation, n (\%) & \\
- Housewife & $26(68.4)$ \\
- Private employee & $6(15.8)$ \\
- Kindergarten teacher & $2(5.3)$ \\
- College student & $2(5.3)$ \\
- Unemployed & $2(5.3)$ \\
Disease duration (months), median (min - max) & $44(1-204)$ \\
MoCA-Ina score, median (min - max) & $25(18-30)$ \\
SLEDAI-2K score, median (min - max) & $6(0-23)$ \\
\hline
\end{tabular}

Note: $\mathrm{SD}=$ Standar Deviation, $\mathrm{n}=$ frequency, $\%=$ percentage 
Table 2.Distribution of cognitive function assessment

\begin{tabular}{ll}
\hline Cognitive function & $\mathrm{n}=38$ \\
\hline Cognitively normal & $18(47.37 \%)$ \\
Cognitively impaired & $20(52.63 \%)$ \\
\hline Impaired cognitive domain & $\mathrm{n}=38$ \\
\hline Visuospatial & $6(15.79 \%)$ \\
Executive function & $26(68.42 \%)$. \\
Attention & $17(44.74 \%)$ \\
Language & $13(34.21 \%)$ \\
Memory & $30(78.95 \%)$ \\
Orientation & $3(7.89 \%)$ \\
\hline
\end{tabular}

Table 3. Distribution of cognitive dysfunction (MoCA-Ina) based on active and inactive SLE (SLEDAI-2K)

\begin{tabular}{lcccc}
\hline & $\mathrm{n}=38$ & \multicolumn{2}{c}{ Cognitive function (MoCA-Ina) } & $\mathrm{p}$ \\
\cline { 3 - 4 } & & $\begin{array}{c}\text { Cognitive dysfunction } \\
\mathrm{n}=20\end{array}$ & $\begin{array}{c}\text { Normal cognitive function } \\
\mathrm{n}=18\end{array}$ & \\
\hline vLEDAlue & & & \\
Active SLE (score $\geq 3)$ & 24 & $13(54.2 \%)$ & $11(45.8 \%)$ & 0.804 \\
Inactive SLE (score $<3)$ & 14 & $7(50.0 \%)$ & $7(50.0 \%)$ & \\
\hline
\end{tabular}

Table 4. Distribution of cognitive dysfunction (MoCA-Ina) in SLE based on severe disease activity and mild to moderate disease activity (SLEDAI-2K)

\begin{tabular}{|c|c|c|c|c|}
\hline & \multirow[b]{2}{*}{$\mathrm{n}=24$} & \multicolumn{2}{|c|}{ Cognitive function (MoCA-Ina) } & \multirow[b]{2}{*}{$\mathrm{p}$ value } \\
\hline & & $\begin{array}{c}\text { Cognitive dysfunction } \\
n=13\end{array}$ & $\begin{array}{l}\text { Normal cognitive function } \\
\qquad n=11\end{array}$ & \\
\hline \multicolumn{5}{|l|}{ SLEDAI-2K } \\
\hline $\begin{array}{l}\text { Severe activity } \\
\text { (score } 12-105 \text { ) }\end{array}$ & 8 & $4(50.0 \%)$ & $4(50.0 \%)$ & \multirow{2}{*}{1.000} \\
\hline $\begin{array}{l}\text { Mild-moderate activity } \\
\text { (skor 3-11) }\end{array}$ & 16 & $9(56.2 \%)$ & 7 (43.8\%) & \\
\hline
\end{tabular}

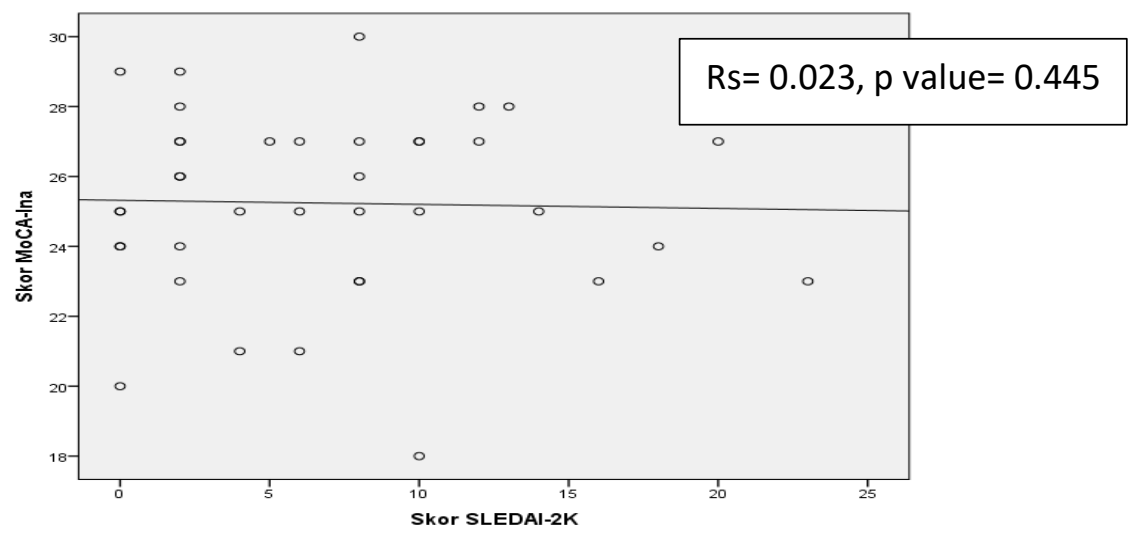

Figure 1. Scatter diagram between SLEDAI-2K score andMoCA-Ina score

Notes: Correlation analysis using Spearman Rank test, significant if $\mathrm{p}<0.05$ 
Table 5. Correlation between age, disease duration, length of education, and cognitive function

\begin{tabular}{|c|c|c|}
\hline & \multicolumn{2}{|c|}{ MoCA-Ina } \\
\hline & Correlation coefficient $\left(\mathrm{r}_{\mathrm{s}}\right)$ & $\mathrm{p}$ value \\
\hline Age & $-0,230$ & 0,083 \\
\hline Disease duration & $-0,035$ & 0,418 \\
\hline Length of education (years) & 0,436 & $0,003^{*}$ \\
\hline
\end{tabular}

Notes: Correlation analysis using Spearman Rank test, significant if $\mathrm{p}<0.05$

\section{DISCUSSION}

Cognitive function test results (Table $\mathbf{1}$ and 2) showed most subjects (52.63\%) had cognitive dysfunction, as memory domain (78.95\%) was the most frequently impaired domain, followed by excecutive function domain (68.42\%). Similar to the study Anjalia et al reported 50\% SLE patients had cognitive dysfunction, most of which (86.67\%) had impaired memory domain. ${ }^{10}$ Adeli et al utilized Mini Mental State Examination (MMSE) as cognitive assessment tool, the results showed that cognitive dysfunction was found three times higher in SLE patients than in non SLE patients with the most frequent impaired domain were orientation and memory domain. ${ }^{22}$

In study that used Phillipines version of MoCA, Allan et al also reported that no correlation between disease activity and cognitive function in SLE. ${ }^{18}$ This finding aligned with study by El-Shafey et al, in which cognitive dysfunction did not correlate with disease activity. ${ }^{19}$ In contrary with our study, Maneeton et al reported there was a correlation between cognitive scores and disease activity at the time of diagnosis was made. However, in this study, Maneeton et al used cognitive score as results from cognitive function assessment by using MMSE, CDT and 5-IADL and the result were calculated with $T$ score. Disease activity was assessed by using MexSledai, as methods and instruments affected study results. ${ }^{20}$ Conti et al published disease activity, anti phospholipid antibody, and chronic damage were associated with cognitive dysfunction in SLE. In that study, cognitive dysfunction was assessed by using Global Cognitive Dysfunction Score (GCDs), while disease activity and chronic damages were assessed by using
SLEDAI-2K and SLICC.21 Different methods and instruments that were used could lead to different results of the studies. There are several screening tools used in assessing cognitive dysfunction in SLE. MoCA and MMSE were the two most common screening tools used in assessing cognitive function, both in clinical practice and research study. In this study, MoCA-Ina was chosen as it has been validated and commonly used as a rapid screening instrument for detecting cognitive dysfunction in Indonesia. It also has higher sensitivity than MMSE, which can detect milder cognitive impairment better than MMSE. Clock Drawing Test (CDT) in MoCA-Ina is an instrument used in cognitive function. More cognitive domains are assessed in MoCAIna than other cognitive function screening tools. 16,17

Table 5 presented correlation analysis by Rank Spearman test between subject's age, disease duration, and length of education with cognitive function based on MoCA-Ina score. Data analysis obtained there was a negative correlation tendency $\left(\mathrm{r}_{\mathrm{s}}=-0.230, \mathrm{p}=0.083\right)$ between subject's age and cognitive function (MoCA-Ina score), this results aligned with previous study which reported cognitive functions, especially attention and memory domain,were affected by subject's age. ${ }^{23}$ Our study showed no correlation between disease duration and cognitive function (MoCA-Ina score). Similarly, Said et al reported there was no correlation between cognitive dysfunction and disease duration in SLE, however this study used Wechsler Memory Scale to assess cognitive dysfunction. ${ }^{24}$ Study by El-Shafey et al. presented contradictive results in which disease duration had a weak correlation with cognitive functions that were assessed by using Trail Making Test (TMT) B (p 
value $=0.049$, with $r$ coefficient $=0.492$ ), yet there was no correlation between disease duration and cognitive function that were assessed by using MoCA test and Trail Making Test (TMT) A. ${ }^{19}$

The study results also showed that there was a moderate positive correlation between subject's length of education and cognitive functions, which were assessed by MoCA-Ina test, suggesting that the longer the length of study was, the higher MoCA-Ina score (better cognitive function) obtained. Consistent with the study, El-Shafey et al. reported subjects who were above12 years old had better cognitive function (higher MoCA score) than those who were under 12 years old ( $p$ value $=0.017$ or $\mathrm{p}<0.05) .19$

\section{CONCLUSION}

There was no correlation between SLE disease activity (SLEDAI 2-K) and cognitive function (MoCA-Ina score). Further investigation regarding the influencing factors are needed.

No disclosure

\section{Acknowledgement}

We would like to acknowledge Dinu Sakti Perwira, MD and Stefanie Yuliana Usman MD for helping preparation for this manuscript. This study is part of 2018 Universitas Padjadjaran's Internal Grant.

\section{REFERENCES}

1. Kasjmir YI, Handono K, Wijaya LK, Hamijoyo L, Albar Z, Kalim H, et al. Indonesian Rheumatology Association Recommendation for Diagnosis and Management of Systemic Lupus Erythematosus.Jakarta: IRA; 2011.p.3-4.

2. Bertsias GC, Boumpas D. In: Bijlsma JW, editor. Systemic lupus erythematosus: pathogenesis and clinical features. EULAR textbook on rheumatic diseases.BMJ; 2012.p.476-505.

3. Guzman J, Cardiel MH, Arce-Salinas A, SanchezGuerrero J, Alarcon-Segovia D. Measurement of disease activity in systemic lupus erythematosus. Prospective validation of 3 clinical indices. The Journal of rheumatology 1992;19(10):1551-8.

4. Liu $Z$, Davidson A. Taming lupus-a new understanding of pathogenesis is leading to clinical advances. Nature medicine 2012;18(6):871-82.

5. Narayanan $\mathrm{CK} \mathrm{MC}$, Shanmuganandan $\mathrm{CK}$, Shankar Gp. Correlation between systemic lupus erythematosus disease activity index, c3, c4 and anti-dsdna antibodies. Med $\mathrm{J}$ Armed Forces India 2010;66:102-7.

6. Freire EAM, Souto LM, Ciconelli RM. Assessment measures in systemic lupus erythematosus. Rev Bras Reumatol 2011;51:70-80.

7. Crow MK, Niewold BT, Kirou KA. Cytokines and interferons in lupus. In: Wallace DJ, Hahn BH, editors. Dubois' lupus erythematosus and related syndromes.8th ed. Philadelphia: Elsevier Saunders; 2013.p. 62-71.

8. Gladman DD, Ibanez D, Urowitz MB. Systemic lupus erythematosus disease activity index 2000. J Rheumatol 2002 Feb;29(2):288-91.

9. Reardon CL. Screening for cognitive impairment.2015 [updated 2016 October 29; cited 2016 Agustus 28]. Available from: http://emedicine.medscape.com/article/1941498overview\#a2.

10. Anjalia S, Ong PA, Atik N, Hamijoyo L. Memory performance in patient with systemic lupus erythematosus using MoCA-Ina in hasan sadikin general hospital bandung. IJR 2017;9(1):25-8.

11. West SG. Clinical aspects of the nervous system. In: Wallace DJ, Hahn BH, editors. Dubois' lupus erythematosus and related syndromes. 8th ed. Philadelphia: Elsevier Saunders; 2013.p. 368-81.

12. Ali II, Savoilane E, Pirzada NA, Tomita M, Santoro TJ. Possible role of the hippocampus in the pathogenesis of cognitive dysfunction in neuropsychiatric systemic lupus erythematosus. Med Hypotheses Res2004;1:111-9

13. Tomita M, Holman BJ, Santoro TJ. Aberrant cytokine gene expression in the hippocampus in murine systemic lupus erythematosus. NeurosciLett2001;302(2-3):129-32.

14. Mak A,Ho RCM, Lau CS. Clinical implications of neuropsychiatric systemic lupus erythematosus. Adv Psychiatr Treat. 2009;15:451-8.

15. Appenzeller S, Cendes F, Costallat LT. Cognitive impairment and employment status in systemic lupus erythematosus: a prospective longitudinal study. Arthritis Rheum2009;61(5):680-7.

16. Husein N, Lumempouw S, Ramli Y, Herqutanto. Montreal cognitive assessment versi indonesia (moca-ina) untuk skrining gangguan fungsi kognitif. Neurona2010;27.

17. Adhikari T, Piatti A, Luggen M. Cognitive dysfunction in SLE: development of a screening tool. Lupus $2011 ; 20(11): 1142-6$.

18. Allan C, Magbitang AT, Hernandez AT, Kenneth, Salido, Evelyn, et al. Assessment of cognitive impairment in systemic lupus erythematosus patients in a rheumatology outpatient clinic of a tertiary government hospital using the mini-mental status exam and the montreal cognitive assessment test-filipino versions.2014 [cited 2016 November 
10]. Available from: http://www.pcp.org.ph/44thannual-convention-abstract-2013-2014.

19. El-Shafey AM, Abd-El-Geleel SM, Soliman ES. Cognitive impairment in non-neuropsychiatric systemic lupus erythematosus. EgyptRheum2012;34(2):67-73.

20. Maneeton B, Maneeton N, Louthrenoo W. Cognitive deficit in patients with systemic lupus erythematosus. Asian Pac J Allergy Immunol 2010;28(1):77-83.

21. Conti F, Alessandri C, Perricone C, Scrivo R, Rezai $\mathrm{S}$, Ceccarelli F, et al. Neurocognitive dysfunction in systemic lupus erythematosus: association with antiphospholipid antibodies, disease activity and chronic damage. PloS one. 2012;7(3):e33824.

22. Mahdavi Adeli A, Haghighi A, Malakouti SK. Prevalence of cognitive disorders in patients with systemic lupus erythromatosus; a cross-sectional study in rasoul-e-akram hospital, tehran, iran. Arch Iran Med 2016;19(4):257-61.

23. Glisky EL. In: Riddle DR, editor.Changes in cognitive function in human aging. brain aging: models, methods, and mechanisms. Boca Raton (FL): CRC Press/Taylor \& Francis Taylor \& Francis Group, LLC.; 2007.

24. Said MSM, Bin Shudim SS, Mohamad K, Shaharir SS, Tong NKC, Ali RA. Subclinical memory dysfunction in malaysian systemic lupus erythematosus patients: association with clinical characteristics and disease activity - a pilot study. Egypt Rheum 2016;38(3):189-94. 